\title{
Protée
}

\section{Images identitaires et rhétorique : la première de couverture de guides touristiques}

\section{Montserrat López Díaz}

Volume 39, numéro 2, automne 2011

URI : https://id.erudit.org/iderudit/1007174ar

DOI : https://doi.org/10.7202/1007174ar

Aller au sommaire du numéro

Éditeur(s)

Département des arts et lettres - Université du Québec à Chicoutimi

ISSN

0300-3523 (imprimé)

1708-2307 (numérique)

Découvrir la revue

Citer cet article

Díaz, M. L. (2011). Images identitaires et rhétorique : la première de couverture de guides touristiques. Protée, 39(2), 113-122. https://doi.org/10.7202/1007174ar
Résumé de l'article

Nous analysons la première de couverture de guides touristiques de la France, de l'Espagne et du Portugal, dans chaque cas à l'intention d'un public exogène. Chacun de ces territoires doit être capable de transmettre une spécificité et de la faire sienne dans l'ambiance absolument concurrentielle de la prolifération de lieux touristiques. C'est ainsi qu'une identité est bâtie à travers un discours fait de mots et surtout d'icônes servant à profiler le pays en question. La première de couverture des guides fournit alors un accès privilégié à une réalité réduite par la pars pro toto à quelques représentations devenues assez familières. On envisagera donc en quoi ces éléments construisent des emblèmes identitaires. 


\title{
IMAGES IDENTITAIRES ET RHÉTORIQUE: LA PREMIÈRE DE COUVERTURE DE GUIDES TOURISTIQUES ${ }^{1}$
}

\author{
MONTSERRAT LÓPEZ DÍAZ
}

L'imagination a fait plus de découvertes que les yeux. (Joubert, 1994: 207)

\section{Introduction}

Il s'agira d'analyser les premières de couverture de guides touristiques de la France, de I'Espagne et du Portugal, dans chaque cas à l'intention d'un public exogène ${ }^{2}$. Les guides touristiques recouvrent aussi bien des aspects de l'organisation du voyage que des informations géographiques, historiques et culturelles, car ils ont tous pour but non seulement de fournir des renseignements pratiques afin de rendre le séjour plus facile et fructueux, mais aussi de faire rêver et $d^{\prime}$ inciter à la découverte de lieux. On demande en effet aux divers types de documents touristiques « des informations clairement présentées et une part d'esthétisme, voire de rêve» (Boyer et Viallon, 1994: 39). Chacun des territoires doit donc être capable de transmettre une spécificité et de la faire sienne dans l'ambiance absolument concurrentielle de la prolifération de lieux touristiques qui règne actuellement non seulement entre des pays géographiquement et culturellement proches, comme c'est le cas dans notre analyse, mais également avec des destinations lointaines.

$C^{\prime}$ est ainsi qu'une identité est bâtie à travers un discours fait de mots et surtout d'icônes servant à profiler la personnalité du pays en question, de manière à le rendre unique et à favoriser sa découverte. Faire rêver et surtout faciliter la conduite, tel est a priori le rôle du guide que nul ne saurait ignorer. Et la première de couverture devra justement rendre l'objet singulier et suffisamment attrayant pour le public; il ne faudrait pas le sous-estimer.
Pays et guides ont besoin de prendre le pas sur la concurrence, car il s'agit de "vendre» non seulement un pays, mais encore et en tout premier lieu un guide. Toutefois, c'est au premier aspect que nous nous intéresserons dans le présent travail ${ }^{3}$.

De ce fait, nous envisagerons en quoi, dans les couvertures, certaines icônes qui apparaissent escortées des noms de pays (France, Portugal et Espagne) ou, le cas échéant, d'autres mots clés, sont représentatives de ces endroits; quelle part elles ont dans la construction d'emblèmes identitaires, c'est-à-dire de figures symboliques, qui bâtissent le lieu et que le lieu fait exister en tant qu'attributs qui lui reviennent immanquablement. Pensons par exemple, dans le cas de la France, que la tour Eiffel, l'Arc de triomphe, Notre-Dame ou le château de Versailles sont a priori plus facilement reconnus dans les médias, comme symbolisant le pays, que la cathédrale d'Amiens ou le château d'Angers. La raison n'est autre que I'habitude de les voir associés. Ce sont autant d'identités, tels des logos, qui restent attachés au lieu dans l'esprit du récepteur.

Notre cadre de réflexion sera sémio-pragmatique: nous interrogerons non seulement les combinaisons des formes et leurs valeurs, le dispositif sémiotique, mais aussi le discours figural et les inférences qui ressortissent à la représentation opérée dans la stratégie de guidage et de captation du lecteur et de signalement des lieux.

\section{Le guide touristique}

Selon le Trésor de la langue française, le guide touristique est « un ouvrage à caractère didactique [qui] aide un touriste à s'orienter, à découvrir les beautés, les curiosités d'une région, $d^{\prime}$ une ville, $d^{\prime}$ un édifice » ${ }^{4}$. Il regorge de renseignements pra- 
tiques, consignes et conseils, mais aussi d'un discours descriptif et incitatif contribuant à rendre le séjour fructueux et agréable pour le plus grand bien du voyageur. Selon Kerbrat-Orecchioni, le guide touristique correspond à un genre hybride qui possède des caractéristiques du style descriptif des récits de voyage et des ouvrages signalétiques à visée purement pratique et informationnelle, mais aussi des discours procéduraux comme les recettes de cuisine, fournissant des recommandations pratiques en vue d'un objectif, des critiques opérant uniquement une discrimination positive et des messages promotionnels (2004: 134-135). Du point de vue axiologique, il arbore un discours positif, car il doit susciter le désir de connaître un endroit. Aussi les réalités problématiques sont-elles occultées ou minimisées, à l'exception, si besoin est, de quelques petits avertissements de rigueur indispensables pour que le touriste ne se méprenne pas ou ne se laisse pas piéger. Mais, dans tous les cas, ce sont les belles images qui priment et, on le sait, le rôle des images est primordial dans le processus cognitif parce qu'elles sont plus facilement mémorisées que le langage. C'est pourquoi on choisit toujours l'angle qui met le plus en valeur l'endroit à visiter ${ }^{5}$.

Le discours du guide touristique correspond ainsi, en grande partie, à celui de la plaquette, que Bonhomme a décrit au moyen de deux procédures: la "schématisation » et la "positivation » (2003 : 14-15). Par la schématisation, on sélectionne, emblématise et rationalise le lieu à travers le choix d'éléments qui deviennent symboliques, en ce qu'ils lui restent attachés dans l'esprit du lecteur, et qui organisent l'espace d'une manière apte à favoriser son exploration, selon une planification stricte et précise et un résultat escompté. Grâce à la positivation, le lieu apparaît valorisé, esthétisé et humanisé: il est par là rehaussé comme un objet exceptionnel, digne d'admiration, combinant art et nature, mais aussi accessible au voyageur prêt à l'explorer dans un esprit hédonique (ibid. : 15-16).

En effet, le guide touristique ${ }^{6}$ doit tout autant attirer l'attention des lecteurs avides de découvertes que combler les attentes des voyageurs en les informant, bien sûr, mais aussi en confirmant ou infirmant leurs idées reçues. De ce fait, le touriste a tendance à chercher sur place la preuve de ce qu'il a déjà vu virtuellement ou ce qu'il a appris lors de ses lectures, conversations, etc. Le guide s'avère de la sorte un intermédiaire entre I'image préconçue que le voyageur a du pays avant sa première visite et celle qu'il trouvera in situ. "C'est dans le guide» ou «ce n'est pas dans le guide» sont presque des mots d'ordre qui astreignent à visiter, à faire ce que le guide dit de visiter ou de faire, le reste n'ayant pas vraiment d'existence.
Comme la une du journal, la première de couverture du guide constitue un aperçu sommaire qui renvoie à des traits essentiels de la réalité "pays $X$ à visiter» dans une optique de loisir ${ }^{7}$, mettant en valeur ce qui mérite d'être vu et connu. Son discours, extrêmement bref, devra ainsi combiner des marques linguistiques et iconiques où rien n'est laissé au hasard, pour engendrer une signification complexe relevant de tout un arsenal sémiotique parfaitement calculé.

La première de couverture du guide possède encore des caractéristiques semblables à celles de la couverture de la brochure, si bien qu'on pourrait très bien lui appliquer les fonctions que Viallon reconnaît à celle-ci, dont les plus importantes seraient: attirer l'attention par rapport à d'autres brochures, éveiller l'intérêt, inciter à la lecture de ce qui suit, résumer ce qui suit, donner une ambiance $(2004: 195)^{8}$. II est évident que, pour cela, il faut opérer un tri qui puisse rendre accessible l'énorme complexité du réel aux destinataires. Par ailleurs, on doit rappeler que, sur la première de couverture, on ne repère pas à proprement parler de traces idiosyncratiques de la nationalité des éventuels destinataires ou de leur couche sociale. Ce type de clivage, quand il existe, ne peut se lire qu'à travers le discours qui figure à l'intérieur du guide.

\section{Rhétorique, sémantique et image}

La réalité "pays $X$ » désignée comme France, Portugal ou Espagne apparaît illustrée d'une ou de plusieurs images qui le réduisent à une partie seulement. La première de couverture des guides fournit alors un accès privilégié à une réalité réduite par la partie pour le tout, pars pro toto, à quelques représentations devenues assez familières.

On dit communément que la synecdoque est une figure de rhétorique ou un procédé linguistique qui consiste à désigner un tout par l'une de ses parties ou vice versa. Mais cette définition un peu trop succincte de la synecdoque est ensuite élargie pour embrasser tout rapport d'inclusion. Elle englobe ainsi la partie pour le tout, mais aussi le plus pour le moins, la matière pour l'objet, l'espèce pour le genre, le singulier pour le pluriel, ou inversement. Par cette stratégie fort habituelle dans le discours courant, on met l'accent sur ce qui s'avère essentiel pour le locuteur.

Bonhomme distingue une synecdoque particularisante, avec des effets focalisants, et une synecdoque généralisante, aux effets amplifiants. La fonction de la synecdoque particularisante est «la fragmentation dénotative» avec deux types de réalisations: « la conversion de la totalité en unité (ou exemplarisation) et 
la conversion de l'unité en partie (ou décomposition)» (2006: 142). La synecdoque généralisante «privilégie par contre les pôles englobants (ensembles ou holonymes) sur les pôles englobés» (ibid. :151).

La synecdoque se distingue de la pars pro parte de la métonymie stricto sensu, où il y a tout simplement contiguïté, et non-inclusion. La métonymie est une figure qui consiste à exprimer un concept par un autre qui lui est uni par une relation nécessaire: I'agent pour le produit, la cause pour l'effet, le contenant pour le contenu, le lieu pour le produit, etc.

Synecdoque vient du grec sun- "ensemble» et ekdokhê «action de comprendre, d'interpréter», ce qui veut dire «interprétation large» (Bacry, 1992: 89). Dans le passé, cette figure n'a pas toujours été décrite comme un trope autonome. Parfois, elle a été incluse dans la métonymie ${ }^{9}$. Bien des stylisticiens et sémanticiens actuels I'incluent encore. Bacry fait de la synecdoque une catégorie de la métonymie, parce que leur fonctionnement est «exactement le même» (ibid. : 89-90). Bonhomme considère également la synecdoque comme un cas particulier de métonymie - un «trope périmétonymique» (2006: 57-58) fondé sur des rapports d'inclusion: "comme la métonymie, la synecdoque crée des transferts de mots et de sens à l'intérieur d'un domaine notionnel »; mais la synecdoque, à la différence de la métonymie, "opère ses transferts entre des notions qui s'intègrent l'une dans l'autre» (1998: 55).

En effet, les deux figures, malgré leurs différences, sont assez proches du point de vue logique. Elles marquent une relation de solidarité entre des entités du même domaine conceptuel, avec une oscillation qui dépend du degré plus ou moins élevé de proximité entre lesdites entités, selon qu'il s'agit d'inclusion ou de contiguïté. Elles ont par ailleurs un comportement syntaxique assez similaire du fait qu'elles remplacent un mot par un autre qui pourrait leur être voisin ou se trouver à proximité sur l'axe syntagmatique.

La question qui se pose pour nous, d'ores et déjà, est de savoir s'il peut y avoir éventuellement synecdoque ou métonymie visuelle. Du reste, il apparaît que les emblèmes des lieux réduisent ceux-ci à quelques représentations succinctes et partielles, mais qui ont le pouvoir de les synthétiser.

\subsection{Figures visuelles?}

Bien des stylisticiens et sémioticiens appliquent, sans s'embarrasser de précautions, certaines figures au domaine visuel - que ce soit aux arts plastiques, à la publicité ou au cinéma - et considèrent qu'on peut parler de figures iconiques tout comme on parle de figures linguistiques. Barthes (1964) ou Durand (1970) se trouvent parmi les premiers à avoir transposé les figures de rhétorique au domaine visuel. La métonymie, se caractérisant par la substitution d'éléments contigus, aurait lieu par exemple dans la publicité quand, à la place d'une voiture, on montre des gens heureux, des paysages ou la route. La synecdoque apparaitrait lorsqu'on présente une partie du produit au lieu du produit lui-même ou l'inverse: ainsi, l'ordinateur peut être remplacé par le clavier, la souris, le bouton de démarrage, ou le contraire.

Ces visualisations se comprennent facilement, bien que les modes de signifier du signe et de l'icône ne soient pas simplement des formes alternatives pour représenter la même chose (Kress et van Leeuwen, 2006: 76). Dans le cas de la métonymie, Bonhomme affirme qu'il est possible d'établir «une certaine homologie cognitivo-interprétative entre les métonymies verbales et ce qu'on pourrait considérer comme des métonymies iconiques», parce qu'elles manifestent des similitudes à un «niveau cognitif préconstruit», sans ignorer toutefois que les codes linguistique et iconique sont différents (2005b : 218-219). Par ailleurs, certaines figures iconiques sont des sources d'interprétations plurielles et trouvent difficilement un équivalent verbal (Bonhomme et Lugrin, 2008: 244-245). De ce fait, il serait impropre de transposer les figures à la lettre. $C^{\prime}$ est ce qu'affirment aussi Kress et van Leeuwen:

Nous ne cherchons pas les (équivalents de) phrases, propositions, noms, verbes, et ainsi de suite, en images. Nous sommes d'avis que les communications linguistique et visuelle peuvent être utilisées toutes les deux pour réaliser les «mêmes » systèmes fondamentaux de signification qui constituent nos cultures, mais chacune le fait à sa manière, différemment et indépendamment.

(2006: 19 ; notre traduction) 10

L'image a certainement une prégnance qui l'écarte de la conceptualisation linguistique. Elle possède une dénotation aux effets référentiels, mais aussi une connotation de nature inférentielle que les théoriciens - quelle que soit la terminologie employée - n'ont pas manqué de souligner. Des enquêtes réalisées auprès du public ont aussi prouvé que la dénotation rigoureuse des icônes est de peu de secours et que leurs significations secondes s'imposent vite. Par ailleurs, on voit dans les objets non pas forcément ce qu'ils sont, mais ce que I'on a appris à y voir.

Les manières spécifiques de signifier de l'image sont les formes, les couleurs, les objets représentés, leur disposition 
sur la page, etc., composant des réalités figurées qu'il faut bien dénommer (Bonhomme, 2005b : 223). Les choix opérés ont le pouvoir de rendre les lieux attrayants.

\section{Premières de couverture, synecdoque et métonymie}

Les mécanismes de substitution de la synecdoque (ou pars pro toto) et de la métonymie (ou pars pro parte) sont au rendezvous dans les premières de couverture des guides touristiques. On désigne et interprète quelque chose par l'intermédiaire de quelque chose d'autre.

On peut considérer que les premières de couverture des trois pays choisis, faisant la part belle aux images, relèvent de la synecdoque iconique particularisante. Celle-ci combinerait décomposition et exemplarisation du fait qu'on se focalise sur une partie d'un endroit. Si nous prenons, par exemple, les couvertures appartenant à la même collection et ayant la même disposition que celle des figures 1, 2 et 3, nous avons une vue sur un village méridional avec sa forteresse (figure 1), un champ de blé, une route et le Mont-Saint-Michel au loin (figure 2), une plage, la mer et des falaises (figure 3), qui constituent des parties de lieux plus vastes et, du coup, des parties de pays. En même temps, ces éléments représentent les pays dans leur totalité et leur pluralité comme des spécimens iconiques exemplaires. On fait effectivement référence à l'ensemble "pays » par une partie de celui-ci qui assume la dénotation du tout. Le tout étant perçu à travers quelques unités types, la charge référentielle qui échoit à celles-ci est considérable. L'image, fragment de village, de monument ou de plage, correspond bien à la structure de la synecdoque, mais relève tout autant de la métonymie si on la considère comme révélatrice de culture, d'architecture, de lieu de repos, d'émerveillement et, par conséquent, comme la cause du tourisme du pays.

Mais analysons ces trois couvertures un peu plus en détail. Elles ont la même disposition puisque chacune est divisée en trois parties. La frange du haut porte le nom du pays en grandes capitales, en rouge pour l'Espagne, en bleu pour la France et en orange pour le Portugal. Ensuite sont affichés les grands visuels. Pour l'Espagne, on montre en contre-plongée le village de Vélez-Blanco ${ }^{11}$ surplombé par son château fort, paysage dénudé de l'Andalousie profonde où règne l'ocre jaune et un peu rougi des maisons et du château lui-même. Le guide de la France exhibe, en contre-plongée également, un champ de blé au premier plan, puis, au-dessus d'un tracé horizontal, s'élève une route avec son alignement d'arbres parfaitement symétriques, sur laquelle circule un tracteur et, au fond, lointaine, se dévoile la silhouette pointue du Mont-Saint-Michel. Dans le cas du Portugal, est présentée, en plongée cette fois, une plage typique de l'Algarve avec ses falaises imposantes qui tantôt la dégagent, tantôt, penchées en avant, défient la mer. Ces trois grandes icônes ont en commun leur caractère spectaculaire.

La partie inférieure des couvertures entremêle la langue et le visuel. Pour l'Espagne, la liste - histoire, plages, églises, plans, tapas, art, villages, fiestas - est agrémentée d'un champ d'oliviers, d'une danseuse andalouse, d'une assiette décorative en céramique et d'un extrait du plan tridimensionnel d'une ville. L'énumération correspondant à la France est restaurants, art galleries, châteaux ${ }^{12}$, wines, cafés, beaches. Le visuel étale un vitrail coloré, une grappe de raisin, la terrasse d'un café avec ses chaises en osier et, encore une fois, le plan d'une ville. Les mots clés pour le Portugal sont monuments, festivals, histoire, plages,
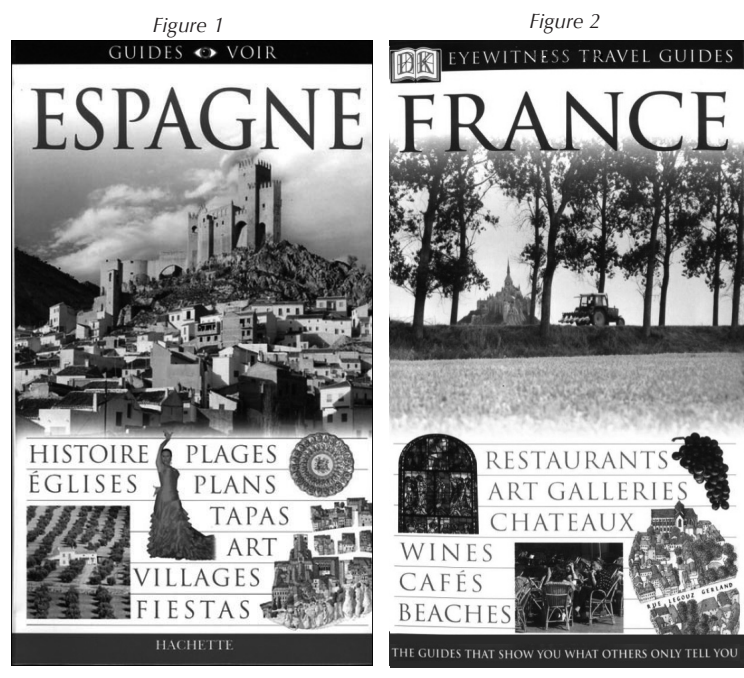

Figure 3

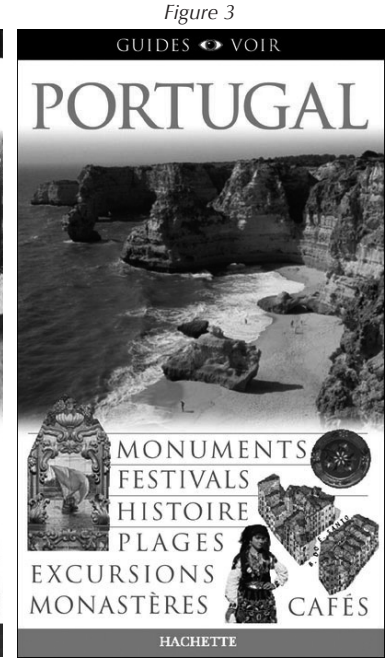

excursions, monastères, cafés; ils se parent d'un carreau de faïence aux tons bleus, d'une assiette en céramique du Minho, d'une femme nazaréenne ${ }^{13}$ au costume traditionnel et, immanquablement, d'un plan.

On le voit, ces couvertures combinent savamment le côté esthétique et le côté utilitaire du guide ${ }^{14}$ : des images qui embellissent et qui illustrent et des propos qui disent ce qui est incontournable dans les trois pays, sans négliger les outils exclusivement pratiques comme les plans, la mention de restaurants, de cafés, etc. 
Si I'on considère maintenant les figures 4 et 5, correspondant respectivement à l'Espagne et à la France et ayant encore les mêmes caractéristiques que les précédentes, on emprunte, dans le cas de l'Espagne, l'image prise en plongée d'une petite ville côtière entourée de fleurs et de la mer. Pour ce qui est de la France, une allée centrale marque un axe de perspective qui débouche sur une église de village et, de part et d'autre, s'ordonnent deux rangées d'arbres. La liste de mots clés dans le cas de l'Espagne est restaurants, museums, fiestas, traditions, cathedrals, tapas, hotels, maps, flamenco, history, beach resorts et apparaît bordée d'un couple de danseurs de flamenco et d'un plan d'une ville. Dans le deuxième cas, on a les mots museums, beaches, châteaux, maps, cafés, history, vineyards, shopping, restaurants, hotels, accompagnés d'une sculpture et d'un plan.

D'ailleurs, on peut aisément remarquer que, dans une optique linguistique - compte tenu également des listes de mots des figures 1 et 2 -, l'identité singulière de la France retombe sur des stéréotypes bien ancrés dans le savoir collectif. Se détachent les châteaux, dont plusieurs sont très célèbres, le vin, dont la France est le premier producteur au monde, et le «shopping», terme générique englobant plusieurs modalités d'achat (y compris les souvenirs indispensables), qui fait sans doute également écho à la mode et au luxe, de la haute couture au prêt-à-porter. L'identité qui se forge pour l'Espagne est axée sur la mention de réalités bien connues, stéréotypées elles aussi: le flamenco, les taureaux 15 et les tapas. La France, I'Espagne ainsi que le Portugal partagent au demeurant la même offre: plages, cuisine, art et monuments.

Le stéréotype rend les choses naturelles: il sert de cadre et de balisage au message choisi, le situant dans les prévisions du récepteur. II passe souvent inaperçu parce qu'il remplit

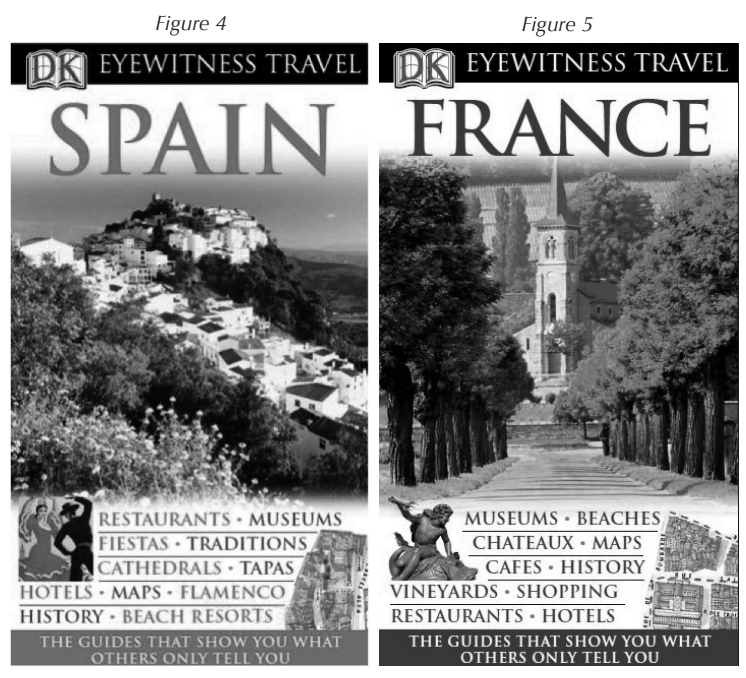

le rôle d'arrière-plan, de préconstruit culturel et de cadre de l'énonciation. Un savoir en amont est ainsi convoqué, dont on ne connaît pas toujours I'origine.

Les quelques éléments de la première de couverture deviennent essentiels dans la mesure où ils doivent supporter, comme approximation préalable à ce que l'on trouvera par la suite - dans le guide d'abord, puis dans le lieu -, le poids de représentation de chaque pays dans son entier et, en même temps, les raisons de sa qualité touristique. De cette façon, «la factualité » du lieu se voit transformée en « une représentation plus ou moins imaginaire» qui ne va pas sans rappeler «le discours épidictique, fondé sur le macro-acte de l'éloge» et "le discours délibératif [...] qui repose sur le macro-acte du conseil »(Bonhomme, 2003 : 13). En effet, dans la première de couverture, on loue un lieu de façon implicite, puisqu'on doit donner envie de le visiter, en complément indispensable au discours didactique du guide montrant le cheminement à suivre. De façon concomitante, on loue aussi le propre guide face aux concurrents, en le rendant attrayant afin de séduire I'acheteur potentiel.

On le voit, les objets apparaissent dans une perspective donnée, sans se laisser scruter complètement, car, en raison de leur caractère illusoire, les photos et les dessins transforment le réel (Joly, 2000: 62). Pour les exemples que nous venons de voir, comme pour ceux que nous verrons par la suite, la composition plastique, dans son harmonie, est fortement inductrice de sens, tout comme la composition chromatique d'ailleurs, où la combinaison de couleurs chaudes (I'ocre du sable et des rochers, des maisons, du château; le jaune et l'orange du champ de blé, des fleurs) et froides (le bleu du ciel et de la mer, le vert des plantes) est savamment calculée pour rendre les espaces attrayants. Nous y reviendrons.

\section{Identité et emblèmes}

Certaines images fonctionnent comme des labels unanimement reconnus, capables de permettre l'identification des villes et des pays à force d'y être liées: la tour Eiffel pour Paris ou la France, le sphinx de Gizeh et les pyramides pour l'Égypte, le Pain de sucre et le Christ Rédempteur pour Rio de Janeiro ou le Brésil, le Parthénon pour Athènes ou la Grèce, etc. Chaque pays a ses icônes qui le symbolisent, parfois sans aucun recours à la langue. Elles ont ainsi le pouvoir de l'identifier quelle que soit la situation de communication. Elles font partie du marketing touristique qui fait exister des lieux en les dotant d'une personnalité (voir Lugrin, 2004 : 245). 
Nous rejoignons Viallon quand il dit que «sans différence, il ne peut pas y avoir de tourisme, c'est l'altérité qui fonde le tourisme [car] la communication touristique est un discours entre identité et altérité » (2004: 193). De ce fait, chaque pays constitue un label, une étiquette, un produit à vendre qui se donne à voir d'emblée sur la première de couverture. C'est pourquoi celle-ci doit lui fournir une identité propre qui aide à le différencier des autres pays.

L'altérité fonde le discours de chaque guide vis-à-vis des autres destinations de la concurrence qui pourraient offrir des expériences semblables, et par rapport au lieu de provenance du lecteur lui-même. Par conséquent, le guide se doit avant tout de créer une identité pour un pays qui par ailleurs, pour le voyageur qui s'y rend, est une altérité qui se matérialise dans le dépaysement qu'il doit éprouver. Mais ce qui est altérité pour l'autre fonde l'identité même du pays célébré: c'est l'identité que le pays en question s'accorde ou souhaite s'accorder à travers des images et des mots clés le symbolisant. Rappelons que le symbole évoque, par une correspondance culturelle, un élément absent. Ainsi, le choix de la couverture est déterminé par ce que le lieu doit devenir aux yeux d'autrui. Son identité se construit donc dans un mouvement dialectique entre la représentation et la réalité, le paraître et l'être.

Or, altérité et identité sont des notions instables, car déjà les natifs sont loin de s'identifier à l'image que l'on donne de leur pays aux étrangers. Dans notre cas, il s'agit d'une identité ou d'une altérité non pas sociologique, mais sémiotique, celle qui se tisse à travers les signes et les icônes en connexion avec l'objet désigné. Chaque endroit a ses spécificités réelles, mais aussi celles bâties dans des discours préalables, de toutes
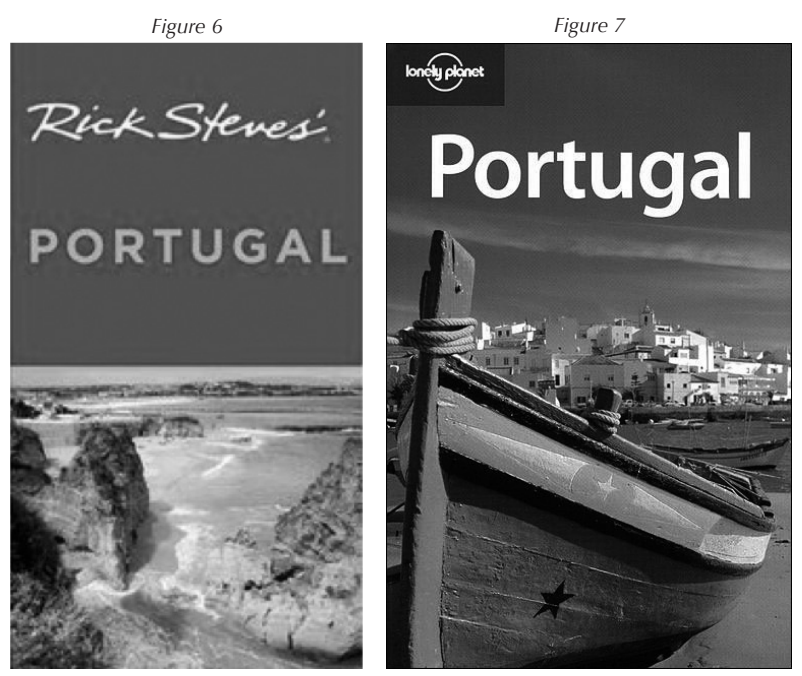

sortes, tenus à son sujet, et qui peuvent converger ou diverger avec d'autres discours ultérieurs. En effet, la mise en discours implique toujours, quel que soit le code, un ancrage cognitif, car des représentations mentales sont mobilisées. Le discours porte en lui-même des traces de ce qui, en amont, le détermine et qui, en aval, le rend compréhensible.

Si I'on observe de près les figures 6,7 et 8 correspondant à des guides du Portugal, I'Algarve est encore souvent à l'honneur. La figure 6 reprend une plage pratiquement déserte encadrée vers le bas afin d'agrandir la part des rochers et réduire celle de la mer. La figure 7 montre un très gros plan d'une barque de pêcheur, derrière laquelle on en voit d'autres sur une petite mer bordée de maisons blanches à tuiles rouges qui ne sauraient être que de la même région. Finalement, et pour une fois, la figure 8 change les décors côtiers paisibles pour un paysage urbain: on peut identifier la ville de Porto avec ses barques sur le Douro au premier plan et, perché sur le haut de la ville, le Palais épiscopal pris en contre-plongée pour mieux mettre en relief son architecture imposante. Il est à signaler que ces premières de couverture n'offrent pas de listes de mots clés ni d'autres illustrations que celles que l'on vient de décrire.

On peut ainsi observer, au moyen des quatre couvertures désignant le Portugal (figures 3, 6, 7 et 8), qu'une grande place est faite aux côtes du sud: images estivales de plages quasiment désertes et d'un village paisible sont au rendez-vous. Le nord du pays est un peu moins représenté avec seulement la ville de Porto. On montre également les carreaux de faïence et la céramique, tout comme la tradition vestimentaire folklorique, ce qui véhicule la richesse culturelle du pays. On note de ce fait une esquisse de diversité dans les représentations, même

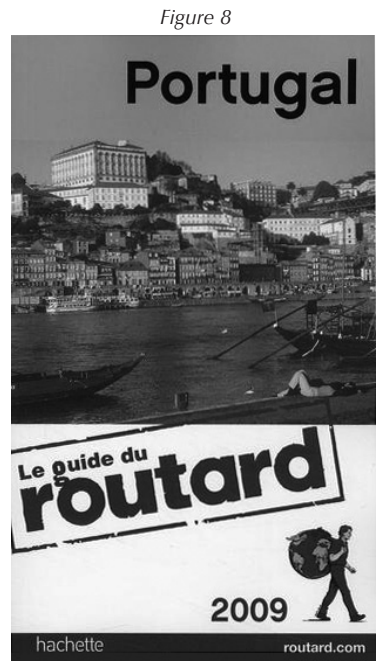

si, d'après les grands visuels, il s'avère difficile de négliger le trio bien connu sun, sea, sand - économie oblige sans doute. La triade en question a été fortement popularisée dans le passé par la publicité, réduisant pendant longtemps certaines destinations du sud de l'Europe presque exclusivement au soleil et à la mer.

Si nous poursuivons notre exploration des couvertures désignant I'Espagne, la figure 9 présente sur fond vert un encadrement au milieu de la page 
qui offre un vaste panorama des moulins à vent et du château de Consuegra ${ }^{16}$, en pleine Manche; plaqué au-dessus, un petit rectangle orange affiche Espagne et, en caractères plus petits, Baléares et Canaries, comme si les deux archipels faisaient bande à part. En dessous de l'encadrement, deux cartouches sont illustrés respectivement par une scène de corrida et des oranges. La figure 10, tout en bleu, étale sur le bas de la page un panneau du taureau d'Osborne ${ }^{17}$ couronnant un champ de tournesols. La prise de vue en contre-plongée met en valeur la superbe silhouette noire du taureau inanimé sur le champ jaune.

Pour finir notre balayage, signalons que, quant à la France, une fois encore I'arrière-pays agricole est donné à voir par un ciel bleu avec ses cultures dessinant des lignes droites, loin du tumulte et des agglomérations. La figure 11 représente une colline plantée de vignes vertes sur laquelle s'élève une église dont le toit du clocher en pointe vient prolonger la verticalité dessinée par les sillons rectilignes des vignobles. On a un ensemble parfaitement symétrique fait d'un mélange d'agriculture et d'architecture. Enfin, la figure 12 reprend le monde paysan, cette fois en Provence: on peut contempler un champ de lavande, avec un gros chêne planté au milieu, où prédominent les lignes droites violettes des sillons.

Si l'on récapitule, on observe dans les douze couvertures une grande diversité de visuels qui rendent difficile l'attribution d'un emblème unique ou fortement stabilisé à chaque pays. En général, les icônes témoignent plutôt d'une personnalité plurielle axée sur la diversité. Le Portugal offre une image assez compacte de «destination soleil » à la réputation bien établie qui mise également sur les traditions et l'art du pays. Pour l'Espagne, les lieux qui apparaissent ne font pas a priori partie du répertoire de lieux facilement identifiables par leur nom, mais se donnent seulement comme des représentants de la géographie andalouse, intérieure ou côtière, et de la Castille, surtout de la Manche. Le panorama des moulins à vent et du château serait à ce sujet l'un des symboles les plus enracinés en raison sans doute de la fiction légendaire de Don Quichotte de la Manche. Le stéréotype du sun, sea, sand comme vitrine de l'Espagne paraît en partie délaissé au profit d'une diversification des attraits du pays ${ }^{18}$, bien qu'on conserve toutefois des visuels emblématiques méridionaux comme les danseurs de flamenco et les corridas. Pour construire l'identité de la France, les grands monuments apparaissent à peine, à l'exception du Mont-Saint-Michel. C'est I'atmosphère des campagnes qui est privilégiée. On voit à travers les différentes couvertures une sorte de tentative de sortir des lieux symboliques fort connus comme Paris, Versailles, la Côte d'Azur ou les châteaux de la Loire. On assiste majoritairement à la visualisation de lieux difficilement identifiables, mais, en même temps, on peut considérer que les couvertures exemplifient l'esprit cartésien qui fait la réputation de la France, et qui se manifesterait dans la vision architecturale de la nature, où le désordre est dompté pour laisser la place à l'ordre et à la perspective. La langue nous le dévoile par ailleurs elle aussi avec ses expressions «jardin français » ou « jardin à la française», qui désignent, comme on le sait, un jardin où les différents éléments sont disposés de façon symétrique. Et les grands espaces de la campagne française de nos couvertures gardent quelque chose de cette symétrie, où rien n'est laissé au hasard, et sont par là même mis en valeur.
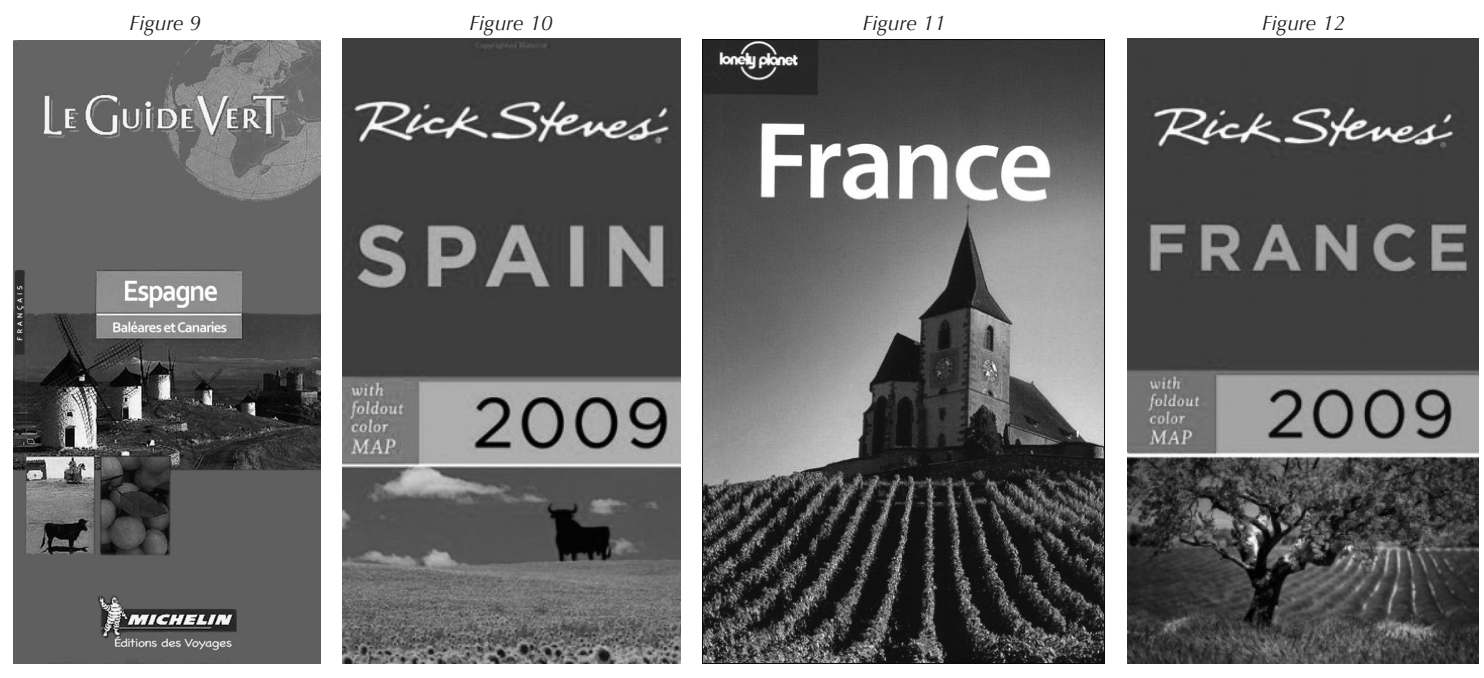
Instruments de la notoriété du pays, les images de la couverture, dans leur naturalité et leur évidence, prévoient une liaison entre deux entités au sein du même domaine référentiel et conceptuel: leur propre valeur désignative comme vue d'un champ de blé et du Mont-Saint-Michel ou d'un champ de lavande et, par extension, leur représentation symbolique du pays. Jouant un rôle essentiel de médiation de la réalité, elles constituent de surcroît, comme on l'a dit, le premier contact avec le lieu à visiter par l'intermédiaire du guide. Aussi doivent-elles être suffisamment séduisantes et symptomatiques.

\section{Analyse sémiotique de trois couvertures}

D'après ce qui vient d'être dit, les couvertures des guides marqueraient une évolution vers la diversité d'emblèmes, tout en gardant des points d'attache à une imagerie plus traditionnelle visible à travers des stéréotypes bien ancrés dans le passé, auxquels, il faut le rappeler, il ne serait ni rentable ni possible d'échapper complètement.

Nous avons choisi les couvertures des figures 6, 10 et 12 en raison de leur relative simplicité, afin d'étudier plus en détail leurs signes visuels ou motifs, dont nous faisons une analyse iconique et iconographique (Cocula et Peyroutet, 1986: 110111) qui tient également compte des résultats d'une enquête ${ }^{19}$.

Du point de vue iconique, chaque couverture est divisée en deux parties: les deux tiers de la page sont en bleu turquoise avec quelques inscriptions et la seconde partie, en bas en forme de rectangle, offre au regard une image du pays. Tout en haut de la page apparaît le nom de l'éditeur du guide en blanc sur fond bleu, Rick Steves'. Puis, presque au centre, est inscrit le nom du pays en capitales jaunes, et plus bas encore on peut voir, dans le cas du Portugal, une seule ligne jaune et, dans celui de l'Espagne et de la France, une bande divisée en deux cartouches. Celui de gauche au fond bleu plus clair contient l'information with foldout color map ( "avec une carte en couleur dépliable») en lettres blanches. Celui de droite montre la date 2009 en caractères noirs sur fond jaune. Finalement, comme on l'a signalé, c'est le dernier tiers de la page qui est illustré. La figure 6 montre, prise en plongée, une crique typique de I'Algarve quasiment déserte: I'eau limpide, la plage de sable fin et les rochers imposants. La figure 10 présente un champ jaune de tournesols, pris en contre-plongée, sans doute en Castille ou en Andalousie. Il est couronné par le taureau d'Osborne et par un ciel bleu avec quelques nuages blancs. Enfin, la figure 12 nous offre, pris en plongée, un champ violet de lavande sillonné avec au milieu un chêne fastueux, qui doit se trouver quelque part en Provence. Cela dit, I'angle de plongée contribue à diminuer l'espace dans les figures 6 et 12, alors que celui de contre-plongée en 10 contribue à l'élargir.

Du point de vue iconographique, I'harmonie chromatique transporte de la légèreté du bleu en haut de la page à la pesanteur des paysages terriens et côtiers en bas. C'est d'ailleurs sur ces paysages que doit s'arrêter le parcours du regard, car l'image, comme on le sait, est très prégnante. Les photographies apparaissent ainsi comme de grandes médiatrices dans la création et la diffusion des destinées touristiques, et exhibent un fragment du réel autour duquel s'édifie un imaginaire l'idéalisant. Par ailleurs, elles s'ajoutent, comme des nouveautés, à d'autres images déjà acquises, ou bien elles reviennent sur des thèmes connus pour les réaffirmer. Mais on montre dans tous les cas des endroits lumineux et paisibles transmettant une sensation de calme et de propreté et possédant par là même tous les atouts pour séduire.

Selon Pastoureau, le bleu est la couleur favorite des Occidentaux (2007: 32), car il invite à la paix et la sécurité. Dans les couvertures, c'est le bleu du ciel qui domine, celui de la lumière, mais $c^{\prime}$ est aussi le bleu de la mer: «Le bleu est la plus profonde des couleurs: le regard $s^{\prime} y$ enfonce sans rencontrer d'obstacle et s'y perd à l'infini, comme devant une perpétuelle dérobade de la couleur » (Chevalier et Gheerbrant, 1973a: 209). C'est pourquoi il incite au rêve et à la rêverie, et par là même à l'évasion. Dans la figure 6, le sable symbolise la recherche du délassement, du plaisir et de la tranquillité:

Le plaisir que l'on éprouve à marcher sur le sable, à s'étendre sur lui, à s'enfoncer dans sa masse souple - qui se manifeste sur les plages - s'apparente inconsciemment au regressus ad uterum des psychanalystes. C'est en effet comme une recherche de repos, de sécurité, de régénération.

(Chevalier et Gheerbrant, 1974d: 135-136).

Ce symbolisme est accentué par la crique entre les deux rochers qui ne peut qu'inviter au calme, à l'isolement et au bonheur dans un espace naturel rétréci. L'espace, en revanche, est plus étendu dans les figures 10 et 12 . Dans la figure 10 apparaît I'un des emblèmes de l'Espagne: le taureau, inerte ici, voit les arènes remplacées par des tournesols, plantes solaires s'il en est. Le jaune est la couleur de la lumière, de la chaleur, du soleil et des vacances, transmettant la joie et l'énergie (Pastoureau, 2007 : 24); il s'oppose au gris du quotidien. Dans la figure 12, le vert de l'arbre est celui du repos terrestre, de la nature et de la liberté. Le chêne, majestueux et protecteur, symbolise par ailleurs I'hospitalité (Chevalier et Gheerbrant, 1973a: 348). Et 
les fleurs violettes de lavande qui I'entourent viennent ajouter au plaisir des yeux celui des arômes, des senteurs de la campagne provençale. Campagne dont la beauté, le calme et le pittoresque ont charmé de grands peintres et écrivains comme Cézanne, Van Gogh, Giono, Pagnol, etc.

Tout bien considéré, malgré leur apparence de relative nouveauté d'espaces naturels maitrisés, en dehors des sentiers battus des formes architecturales monumentales, ces illustrations que la nature offre au regard sont elles aussi assez stéréotypées. En effet, elles renvoient encore à des attributs que l'on connaît bien pour les trois pays: métonymie de la plage et de la mer pour la baignade au Portugal; celle des tournesols pour le soleil et du taureau pour les corridas en Espagne; et celle des fleurs de lavande pour les senteurs embaumées de Provence ou les parfums et les soins de beauté en France - ceux-ci en principe plus directement liés au commerce français qu'au tourisme proprement dit. Mais il est vrai que, pour l'invitation au voyage et aux vacances, si l'image de la côte du sud du Portugal est familière, ainsi que celle du taureau pour l'Espagne, les tournesols sont moins représentatifs de ce pays-là, ou encore peut-être le champ de lavande pour l'image extérieure du tourisme de la France ${ }^{20}$, quoiqu'il soit typique de Provence et que celle-ci soit très touristique.

\section{Conclusion}

Les médias, y compris Internet, jouent un rôle majeur dans la construction et la circulation des images identitaires qui popularisent les lieux définitoires et emblématiques d'un pays. L'image rêvée, créée par les visuels des premières de couverture, se voit par ailleurs prolongée grâce aux cartes postales et aux photos souvenirs (voir Lanquart et Hollier, 1981 : 73). Ces icônes fonctionnent comme des prototypes, de bons représentants de la catégorie, mais également comme des stéréotypes du fait que la répétition les rend familiers. Aussi ces portions de réel en simplifient-elles la complexité, réduisant une perception large et changeante à quelques traits extrêmement schématisés, qui s'avèrent essentiels à la compréhension.

Les reproductions iconiques sont non pas simples ni autonomes, mais bien subordonnées à leur contexte, dans ce cas leur support, c'est-à-dire la couverture du guide qui fait qu'on les interprète comme des outils de promotion d'un lieu. Les informations «contenues» dans la première de couverture du guide touristique sont ainsi utilisées par le lecteur pour rétablir d'autres informations qui manquent et, en même temps, il est capable d'anticiper la suite.
Si les figures de rhétorique ont la fonction de rendre les productions linguistiques plus efficaces, il en est de même pour les icônes, qui sont fondamentales dans les stratégies de marketing, car elles peuvent rendre attirant ce qui en réalité pourrait être plutôt fade ou anonymisé. À travers les images photographiques, on a l'impression d'une grande vérité, «d'y être» en somme, ce qui peut aussi rendre le message «dangereux pour celui qui n'a pas les moyens de le dominer» (Cocula et Peyroutet, 1986: 26). Cela est assez fréquent dans l'information médiatique. On sait, en effet, que les images tout comme les mots d'ailleurs ont un énorme pouvoir de manipulation.

Il nous semble qu'un travail diachronique serait avantageux, afin de voir de près une possible évolution dans les premières de couverture des guides touristiques. Une évolution qui, a priori, pourrait être analogue à celle subie au fil des ans par les premières de couverture des manuels de langue étrangère par exemple.

\section{NOTES}

1. Travail partiellement financé par le projet PR2009/0499 du ministère de l'Éducation espagnol et réalisé à l'Université de Berne. Une version préalable de cette recherche a fait l'objet d'un exposé au IIe Congrès luso-espagnol d'études francophones qui s'est tenu à Barcelone du 21 au 23 octobre 2009. Je remercie Manuel Bruña, Rosa M. Chaves et José D. de Almeida pour leurs précisions concernant l'identification de certaines représentations photographiques.

2. Au total, douze couvertures ont été prélevées (4 par pays concerné) sur le site commercial Amazon spécialisé dans la vente de livres (www. amazon.com) en octobre 2009. Elles appartiennent aux guides suivants: Guide du Routard, Guide Vert, Lonely Planet, Rick Steves', Eyewitness Travel et Guides Voir. Étant donné que sur ce site sont disponibles seulement des ouvrages en anglais et en français, les guides de la France sont en anglais, et ceux de l'Espagne et du Portugal en anglais et en français. On a veillé ainsi au fait que les publications soient toujours destinées à un public étranger.

3. Chaque éditeur ou chaque collection montre sa propre idée de ce que doit être un guide. Mais, bien qu'elles soient conçues différemment, il y a une convergence certaine dans les différentes couvertures que nous analysons, en ce qu'elles visent à offrir tout l'éclat du pays. C'est le discours que l'on trouve à l'intérieur du guide qui fait la différence, le cas échéant, selon le public auquel il est destiné.

4. En ligne: http://atilf.atilf.fr/tlf.htm (page consultée le 29 avril 2011). 5. «La médiocrité desservirait le guide», affirment Lanquart et Hollier (1981 : 62). II n'est pas question, par exemple, de montrer le quartier industriel ou marginal d'une ville; bien au contraire, on donne à voir des espaces rassurants où tout est ordonné et maîtrisé.

6. C'est l'imprimeur lyonnais Charles Estienne qui, en 1552, publia les deux premiers guides français: La Guide [sic] des chemins de France et Les Fleuves du royaume de France (Boyer et Viallon, 1994 : 54-55). 
7. «Le client potentie/ du tourisme est d'abord celui qui rêve de départ, d'une destination, d'une activité ludique» (Boyer et Viallon, 1994: 4). 8.Il semble s'être inspiré en partie du modèle AIDA de la publicité: attention, intérêt, désir, achat.

9. Par exemple chez Lamy, Le Guern, Ruwet (voir Bonhomme, 2005a: 45-46); ou encore Ullmann, Jakobson (voir Nyckees, 1998: 102).

10. «We do not look for (the analogues of) sentences, clauses, nouns, verbs, and so on, in images. We take the view that language and visual communication can both be used to realize the "same" fundamental systems of meaning that constitue our cultures, but that each does so by means of its own specific forms, does so differently, and independently».

11. Il se trouve dans la province d'Almería.

12. En guise d'authenticité et d'idiosyncrasie, on rend hommage à la langue du pays concerné. On a ainsi, pour la France, châteaux, cafés dans la liste de mots en anglais, et, pour l'Espagne, tapas, fiestas, flamenco dans les listes en français et en anglais, même si lesdits mots circulent habituellement dans les langues emprunteuses.

13. Nazaré est une ville qui se trouve dans le « district» de Leiria. 14. La succession de mots clés saupoudrés de petites images est exclusive de la collection Eyewitness Travel Guides (version anglaise) et Guides Voir d'Hachette (version française).

15. Fiesta est un mot polysémique qui renvoie à la fiesta nacional, c'està-dire les "corridas de taureaux», mais aussi à des kermesses populaires et des festivités traditionnelles.

16. Consuegra se trouve dans la province de Tolède.

17. Ce taureau est une silhouette conçue à l'origine par legroupe Osborne comme panneau pour la publicité d'un de ses spiritueux. Maintenant, il ne porte plus de marque, bien que tout Espagnol soit capable de l'y associer, et est devenu presque une icône obligée du label Espagne. II se trouve bien visible sur des collines, le long de routes très fréquentées. 18. Il semblerait que certains produits touristiques devant convenir perpétuellement à la clientèle ont « une vie » et « une mort » (Lanquart et Hollier, 1981:20); raison pour laquelle les campagnes publicitaires axées sur le soleil et la mer appartiennent au passé dans le cas de l'Espagne. Cela ne serait pas dû à la lassitude, mais à un besoin de se différencier des autres pays méridionaux qui peuvent, eux aussi, offrir des plages. À ce sujet, on peut consulter le document La Stratégie touristique de I'Espagne. En ligne: http://www.tourismes.info/dossiers/IREST_Espagne. pdf (page consultée le 29 avril 2011).

19. Enquête réalisée auprès d'une trentaine d'étudiants universitaires de lettres. Deux questions leur ont été posées: 1) Que voyez-vous? et 2) Comment interprétez-vous ce que vous voyez?

20. Tout au moins, les enquêtes que nous avons effectuées l'attestent.

\section{RÉFÉRENCES BIBLIOGRAPHIQUES}

BACRY, P. [1992] : Les Figures de style, Paris, Belin.

BAIDER, F., M. BURGer et D. GOutsos (dir.) [2004] : La Communication touristique, Paris, L'Harmattan.

BARTHES, R. [1964] : "Rhétorique de l'image», Communications, no 4, 40-51.

Bonhomme, M. [1998] : Les Figures de style, Paris, Seuil ;

[2003] : «L'éloge de la ville: rhétorique d'une plaquette touristique sur Berne», dans P. Marillaud et R. Gauthier, Les Langages de la ville, Toulouse, CALS, 13-21;

[2005a] : Pragmatique des figures, Paris, Champion;

[2005b] : "Peut-on parler de métonymie iconique? », dans

S. Badir, et J.-M. Klinkenberg, Figures de la figure. Sémiotique et rhétorique générales, Limoges, Pulim, 215-228;

- [2006] : Le Discours métonymique, Berne, Peter Lang.

BONHOMME, M. et G. LUGRIN [2008] : «Interprétation et effets des figures visuelles dans la communication publicitaire: le cas de la métonymie et de la métaphore », Studies in Communication Sciences, vol. 8, no 1, 237-258.

BOYER, M. et P. VIALLON [1994]: La Communication touristique, Paris, PUF. BuFFON, B. [2002] : La Parole persuasive, Paris, PUF.

Chevalier, J. et A. Gheerbrant [1973a, b; 1974c, d] : Dictionnaire des symboles, 4 vol., Paris, Seghers.

Cocula, B. et C. Peyroutet [1986] : Sémantique de l'image, Paris, Delagrave.

DURAND, J. [1970] : "Rhétorique et image publicitaire», Communications, nO15, 70-95.

Gervereau, L. [2000] : Les images qui mentent. Histoire du visuel au Xxe siècle, Paris, Seuil.

Houdebine, A.-M. [1994] : "Convergence. Divergence. Périphérie», Travaux de linguistique et de sémiologie, no 5-6, 47-51.

JOLY, M. [(1994) 2000] : L'Image et les Signes, Paris, Nathan/HER.

JOUBERT, J. [1994] : Carnets, tome 1, Paris, Gallimard.

Kerbrat-OreCChIONI, C. [2004] : "Suivez le guide! Les modalités de I'invitation au voyage dans les guides touristiques: I'exemple de l' "île d'Aphrodite" », dans F. Baider, M. Burger et D. Goutsos (dir.), 133-150. KRESS, G. et T. van LEEUWEN [(1996) 2006] : Reading Images. The Grammar of Visual Design, Londres, Routledge.

LANQUART, R. et R. HOLLIER [1981] : Le Marketing touristique, Paris, PUF. LÓPEZ DÍAZ, M. [2002] : "L'interdépendance du texte et de l'illustration ou comment la publicité forge des histoires", Semiotica, vol. 142, no 1-4, 125-152;

- [2004] : «La stéréotypie culturelle dans I'enseignement-apprentissage du FLE », Bulletin de l'association des professeurs de français de Galice, no1, 73-89.

LUGRIN, G. [2004] : «La construction des icônes identitaires des lieux touristiques: Chypre dans les publicités touristiques de langue française», dans F. Baider, M. Burger et D. Goutsos (dir.), 151-172.

NYCKEES, V. [1998] : La Sémantique, Paris, Belin.

PASTOUREAU, M. [2007] : Dictionnaire des couleurs de notre temps. Symbolique et société, trad. par N. Petit, Paris, Bonneton.

VIALLON, P. [2004] : "La Méditerranée au risque de la communication touristique», dans F. Baider, M. Burger et D. Goutsos (dir.), 191-214. 